\title{
Coherent single-photon absorption by single emitters coupled to one-dimensional nanophotonic waveguides
}

\author{
Chen, Yuntian; Wubs, Martijn; Mørk, Jesper; Koenderink, A. Femius
}

Published in:

New Journal of Physics

Link to article, DOI:

10.1088/1367-2630/13/10/103010

Publication date:

2011

Document Version

Publisher's PDF, also known as Version of record

Link back to DTU Orbit

Citation (APA):

Chen, Y., Wubs, M., Mørk, J., \& Koenderink, A. F. (2011). Coherent single-photon absorption by single emitters coupled to one-dimensional nanophotonic waveguides. New Journal of Physics, 13(10), 103010.

https://doi.org/10.1088/1367-2630/13/10/103010

\section{General rights}

Copyright and moral rights for the publications made accessible in the public portal are retained by the authors and/or other copyright owners and it is a condition of accessing publications that users recognise and abide by the legal requirements associated with these rights.

- Users may download and print one copy of any publication from the public portal for the purpose of private study or research.

- You may not further distribute the material or use it for any profit-making activity or commercial gain

- You may freely distribute the URL identifying the publication in the public portal 
Coherent single-photon absorption by single emitters coupled to one-dimensional nanophotonic waveguides

This article has been downloaded from IOPscience. Please scroll down to see the full text article.

2011 New J. Phys. 13103010

(http://iopscience.iop.org/1367-2630/13/10/103010)

View the table of contents for this issue, or go to the journal homepage for more

Download details:

IP Address: 192.38.67.112

The article was downloaded on 14/10/2011 at 08:04

Please note that terms and conditions apply. 


\title{
Coherent single-photon absorption by single emitters coupled to one-dimensional nanophotonic waveguides
}

\author{
Yuntian Chen $^{1,3}$, Martijn Wubs ${ }^{1}$, Jesper Mørk ${ }^{1}$ \\ and A Femius Koenderink ${ }^{2}$ \\ ${ }^{1}$ DTU Fotonik, Department of Photonics Engineering, Ørsteds Plads, \\ DK-2800 Kgs Lyngby, Denmark \\ ${ }^{2}$ Center for Nanophotonics, FOM Institute for Atomic and Molecular Physics \\ (AMOLF), Science Park 104, 1098 XG Amsterdam, The Netherlands \\ E-mail: yche@fotonik.dtu.dk \\ New Journal of Physics 13 (2011) 103010 (21pp) \\ Received 28 June 2011 \\ Published 11 October 2011 \\ Online at http://www.njp.org/ \\ doi:10.1088/1367-2630/13/10/103010
}

\begin{abstract}
We study the dynamics of single-photon absorption by a single emitter coupled to a one-dimensional waveguide that simultaneously provides channels for spontaneous emission (SE) decay and a channel for the input photon. We have developed a time-dependent theory that allows us to specify any input single-photon wavepacket guided by the waveguide as the initial condition, and calculate the excitation probability of the emitter, as well as the time evolution of the transmitted and reflected fields. For single-photon wavepackets with a Gaussian spectrum and temporal shape, we obtain analytical solutions for the dynamics of absorption, with maximum atomic excitation $\sim 40 \%$. We furthermore propose a terminated waveguide to aid the single-photon absorption. We found that for an emitter placed at an optimal distance from the termination, the maximum atomic excitation due to an incident single-photon wavepacket can exceed $70 \%$. This high value is a direct consequence of the high SE $\beta$-factor for emission into the waveguide. Finally, we have also explored whether waveguide dispersion could aid single-photon absorption by pulse shaping. For a Gaussian input wavepacket, we found that the absorption efficiency can be improved by
\end{abstract}

\footnotetext{
${ }^{3}$ Author to whom any correspondence should be addressed.
} 
a further $4 \%$ by engineering the dispersion. Efficient single-photon absorption by a single emitter has potential applications in quantum communication and quantum computation.

\section{Contents}

1. Introduction 2

2. Model 4

2.1. Hamiltonian, initial conditions and equations of motion . . . . . . . . . 5

2.2. Dynamics of absorption, reflection and transmission in one-dimensional (1D) and quasi-1D waveguides . . . . . . . . . . . . . 7

3. Gaussian pulse excitation of a 1D waveguide $\quad 8$

3.1. Stationary limits of the single-photon absorption in a 1D waveguide . . . . . 10

3.2. Time dependence of the transmittance and reflectance of the single-photon wavepacket in a $1 \mathrm{D}$ waveguide . . . . . . . . . . . . . . . . 12

4. Gaussian input on a semi-infinite 1D waveguide $\quad 13$

4.1. Enhanced spontaneous emission (SE) and the SE $\beta$-factor by a terminated metallic nanowire . . . . . . . . . . . . . . . . . . 13

4.2. Single-photon wavepacket propagation along the terminated metallic nanowire 15

4.3. Enhanced single-photon absorption by a terminated metallic nanowire . . . . . 16

5. Conclusion 17

Acknowledgments $\quad 18$

Appendix A. Normalization in the continuous-mode quantization scheme $\quad 18$

Appendix B. Laplace transform method for a Gaussian input single-photon wavepacket $\quad 19$

$\begin{array}{ll}\text { References } & 20\end{array}$

\section{Introduction}

Ultimate control over single light quanta, the emission of single photons, the absorption of single photons and the routing of photons between qubits is of core interest for quantum information technology and is extensively studied in [1-9]. Ideally, one could use single photons that rarely interact with each other and the environment as natural messengers of quantum information between nodes, where actual operations take place. Such nodes could then consist of localized atoms or quantum dots that can interact strongly with each other or external stimuli via, e.g., sideband coupling, radio-frequency (RF) fields or electrical gate signals in an efficient and controllable way. Thus, it is extremely desirable to map a flying photonic qubit state onto an atomic qubit with unit probability [10,11]. However, such interfacing between an atom qubit and a flying photonic qubit is challenging, since it requires simultaneously an open photonic system for easy interfacing with freely propagating photons, yet also a high light-matter interaction strength, which is usually associated with the use of a high$Q$, closed photonic system surrounding the atomic qubit. In the absence of a high- $Q$ cavity, one can either use a large ensemble of atoms to compensate for the weak optical transition strengths of single atoms [12] or use highly focused optical beams to excite single atoms or molecules as efficiently as possible [13-15]. Among different mapping techniques, direct 

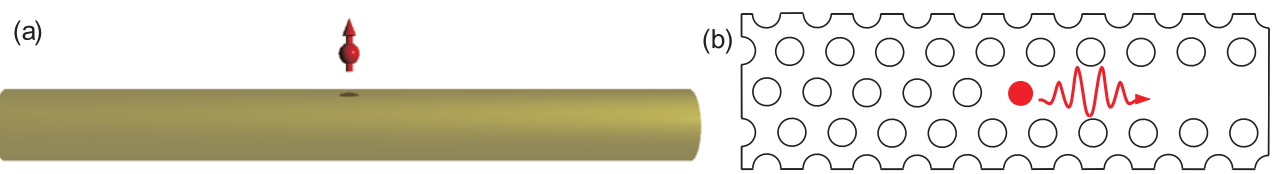

(c)

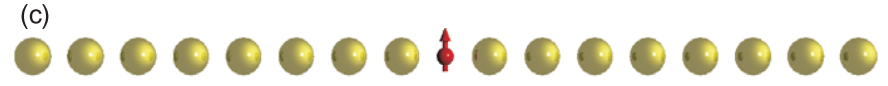

Figure 1. Possible experimental realizations of a 1D waveguide strongly coupled to a single emitter: (a) a propagating surface plasmon polariton (SPP) mode on a metallic nanowire, (b) a guided mode in a photonic crystal waveguide and (c) a guided plasmonic mode on an infinitely long plasmon particle chain.

absorption of single photons by light emitters is an attractive option that may be realized using recent advances in the engineering of complex photonic environments [13, 16-23]. Such efficient single-photon absorption is not only of relevance for quantum optics, circuit quantum electrodynamics (QED) [24, 25] and the few-photon community [7-9, 26], but is also of central relevance for detectors, photovoltaics, optical sensing and microscopy based on absorption or fluorescence $[27,28]$.

Recent advances in nanophotonics enable the funneling of almost all the single photons emitted by a single emitter into a single mode $[29,30]$ or directing single photons into narrow beams [31-33] and even dynamical steering of singe-photon emission [34]. As sketched in figure 1, it has been shown that these one-dimensional (1D) or quasi-1D waveguides can be used to efficiently control the spontaneous emission (SE) [29, 30, 35-38]. Inversely, reciprocity in classical electrodynamics predicts efficient coupling of single photons with an emitter, provided the incoming single-photon wavepacket is fed through the channel which is associated with a high SE rate. Recent theoretical work [20, 22, 39-41] has hence focused on the interaction of single emitters and single photons guided by $1 \mathrm{D}$ waveguides. However, this work mainly concerns the reflection and the transmission probability of single-photon wavepackets of very narrow spectral bandwidth interacting with a single emitter. These models consist of stationary solutions for the interaction of a two-level system with a 1D waveguide. In such stationary cases, the photon wavepackets have a quasi-infinite temporal extent, and hence the atom is essentially in the ground state at all times. In this case, the atom can simply be treated as a point scatterer $[22,39,42]$. To optimize the atomic excitation probability, a time-dependent treatment is required to predict which photon wavepackets have optimal temporal and spatial profiles for atomic excitation. Originally it was pointed out by Cirac et al [11, 43] that a timereversal symmetric photon wavepacket can be used to efficiently transfer quantum states among distant nodes consisting of a $\Lambda$-type atomic medium. The interesting concept of time-reversal symmetry was also applied to two-level atoms, showing that it is indeed possible to perfectly invert an atomic qubit using photon wavepackets that are spatially and temporally the inverse of the photon wavepacket emitted by a qubit through SE [23, 44]. However, realizing this prediction requires highly nontrivial pulse shaping, especially at the single-photon level [45]. Thus, there is a large need to evaluate the time evolution of atomic excitation efficiencies upon excitation by photon packages of practically realizable temporal shape in realistic nanophotonic geometries.

Several routes are potentially feasible to evaluate the time evolution of atomic excitation efficiencies in practical geometries. For instance, one might first find the stationary interacting 
eigenstates of the photon-atom system, project an incident condition on those states and subsequently apply the time evolution operator. This extension of stationary theory was applied very recently by Rephaeli et al [44] to derive the perfect inverting pulse. In this paper, we implement an alternative route. In our complementary model, we develop a theory directly in the time domain that does not require the interacting eigenstates to be found. Our timedependent theory quantifies what atomic excitation efficiencies can be reached using practically achievable single-photon wavepackets in 1D waveguides. As sketched in figure 1, we consider a single-photon wavepacket propagating along a 1D waveguide, interacting with a single emitter. We explore the possibility of maximizing the single-photon absorption by the emitter via engineering the photonic environment of the emitter and by shaping the pulse of the input single-photon wavepacket through waveguide dispersion. We study 1D or quasi-1D waveguides, considering all the SE channels, since it is the competition between the pumping channel and the SE channel of the emitter that will ultimately determine the absorption efficiency. We focus on the role of the temporal coherence of the input single-photon wavepacket in exciting the emitter in the $1 \mathrm{D}$ waveguide. The formalism can be generalized to treat 3D light-scattering problems for examining the role of spatial coherence, which will be addressed in a future paper.

This paper is organized as follows. In section 2, we formulate the model and derive general solutions for the dynamics. Section 3 presents the results obtained for Gaussian input single-photon wavepackets propagating along an infinitely long 1D waveguide. We obtain an analytical solution for the dynamics of atomic excitation. Furthermore, we present a physically transparent model that relates our dynamical model to the stationary reflection and transmission spectrum, and also present a simple model for the time-dependent reflection and transmission. We found that the maximum absorption probability depends strongly on the temporal coherence of the input single-photon wavepacket. In section 4 , we consider an emitter located near the termination of a half-truncated 1D waveguide. Near such a termination, the SE $\beta$-factor for unidirectional emission can be strongly enhanced. By reciprocity, uni-directional emission with a high $\beta$-factor also results in the highest possible atomic excitation probability. Our work thus provides a simple route to optimize photonic waveguide structures for photon-qubit interaction. Finally, we investigate the possibility of improving the atomic excitation by pulse chirping or pulse dispersion to modify the temporal coherence. In section 5, we conclude the paper.

\section{Model}

In this section, we will outline our theory for calculating the dynamics of single-photon absorption. Our model follows a similar procedure to that employed in [46-48]. Dorner and Zoller [46] investigated the SE impacted by a mirror within the framework of a 1D model using a non-Markovian treatment, which recovers several features beyond the Markovian limit. In [47], the authors showed dissipative features induced by bringing an atom into a waveguide system, namely significant scattering induced by the atom of the incoming light wavepacket, as well as demonstrating the low likelihood of population transfer due to a large damping rate in their system. Kien et al [48] further showed that in the vicinity of the surface of a dielectric nanofiber, the transmittance of the field in the stationary regime can be substantially reduced due to scattering into radiation modes and backward-guided modes. The difference between our work and those in the literature is the following: [46] concerns SE and resonance fluorescence under non-Markovian conditions, while [47, 48] focus on the light scattering properties mediated by a single emitter coupled to a 1D waveguide. Our work is devoted 
to maximizing the efficiency of single-photon absorption by a single emitter coupled to a nanophotonic environment. In section 2.1, we construct a general model for single-photon absorption valid for arbitrary (nonabsorbing) 3D photonic structures with arbitrarily shaped input wavepackets. In section 2.2, we apply the general model to the study of the dynamics of single-photon absorption by a single atom coupled to a $1 \mathrm{D}$ waveguide $[22,39]$. The reflection and transmission coefficients derived in stationary theories can be extracted by evaluating our time-dependent solutions at times far later than the time interval where the photon wavepacket collides with the atom, and after the emitter has relaxed back into the ground state due to SE.

\subsection{Hamiltonian, initial conditions and equations of motion}

In a finite volume the electromagnetic field can be decomposed into discrete modes. In the discrete-mode quantization scheme, the interaction between a single-photon wavepacket and the emitter is modeled by the Hamiltonian

$$
\hat{H}_{\text {tot }}=\hat{H}_{0}+\hat{H}_{\mathrm{I}},
$$

where $\hat{H}_{0}$ defines the free constituents $\hat{H}_{0}=\hbar \omega_{0}|e\rangle\langle e|+\sum_{\lambda} \hbar \omega_{\lambda} \hat{a}^{\dagger}(\lambda) \hat{a}(\lambda)$ and $\hat{H}_{\mathrm{I}}$ defines the interaction part $\hat{H}_{\mathrm{I}}=\sum_{\lambda} \hbar\left[g_{\lambda} \hat{a}(\lambda)|e\rangle\left\langle g\left|+g_{\lambda}^{*} \hat{a}^{\dagger}(\lambda)\right| g\right\rangle\langle e|\right]$. Here $|g\rangle$ and $|e\rangle$ represent the atom ground and excited states, respectively, $\omega_{0}$ denotes the atomic transition frequency and $\hat{a}(\lambda)$ is the annihilation operator for mode $\lambda$. Without loss of generality, we take the emitter position as our real-space origin (the emitter located at $\mathbf{r}=0$ ). We expand the Schrödinger-picture state $|\psi(t)\rangle$ at time $t$ in the basis of all states with one excitation, i.e. $|e, 0\rangle,\left|g, 1_{\lambda}\right\rangle$, as follows:

$$
|\psi(t)\rangle=C_{0}^{\mathrm{e}}(t) \mathrm{e}^{-\mathrm{i} \omega_{0} t}|e, 0\rangle+\sum_{\lambda} \mathrm{e}^{-\mathrm{i} \omega_{\lambda} t} C_{\lambda}^{\mathrm{g}}(t)\left|g, 1_{\lambda}\right\rangle .
$$

By separating the time dependence driven by $H_{0}$, the coefficients $C_{0}^{\mathrm{e}}(t)$ and $C_{\lambda}^{g}(t)$ are essentially solved in the interaction picture. Since we are interested in the absorption of a single-photon wavepacket, we assume that in the initial state, the atom is unexcited $\left(C_{0}^{\mathrm{e}}(0)=0\right)$, while the initial wavepacket is described by $C_{\lambda}^{\mathrm{g}}(0)=\xi(\lambda)$.

The equations of motion are

$$
\begin{aligned}
& \mathrm{i} \hbar \frac{\mathrm{d} C_{0}^{\mathrm{e}}(t)}{\mathrm{d} t}=\sum_{\lambda} C_{\lambda}^{\mathrm{g}}(t)\left\langle e, 0\left|H_{I}\right| g, 1_{\lambda}\right\rangle \mathrm{e}^{-\mathrm{i}\left(\omega_{\lambda}-\omega_{0}\right) t}, \\
& \mathrm{i} \hbar \frac{\mathrm{d} C_{\lambda}^{\mathrm{g}}(t)}{\mathrm{d} t}=C_{0}^{\mathrm{e}}(t)\left\langle g, 1_{\lambda}\left|H_{I}\right| e, 0\right\rangle \mathrm{e}^{\mathrm{i}\left(\omega_{\lambda}-\omega_{0}\right) t} .
\end{aligned}
$$

The transition matrix element $\left\langle g, 1_{\lambda}\left|H_{I}\right| e, 0\right\rangle$ can be simplified as $\left\langle g, 1_{\lambda}\left|H_{\mathrm{I}}\right| e, 0\right\rangle=\hbar g_{\lambda}^{*}$, where $g_{\lambda}$ is the well-known interaction strength of a single emitter with mode $\lambda$ [51]. Integrating equation (4) yields

$$
C_{\lambda}^{\mathrm{g}}(t)=C_{\lambda}^{\mathrm{g}}(0)-\mathrm{i} \int_{0}^{t} C_{0}^{\mathrm{e}}\left(t^{\prime}\right) g_{\lambda}^{*} \mathrm{e}^{\mathrm{i}\left(\omega_{\lambda}-\omega_{0}\right) t^{\prime}} \mathrm{d} t^{\prime},
$$

and using this in equation (3), one can obtain

$$
\frac{\mathrm{d} C_{0}^{\mathrm{e}}(t)}{\mathrm{d} t}=-\mathrm{i} \sum_{\lambda} C_{\lambda}^{g}(0) g_{\lambda} \mathrm{e}^{-\mathrm{i}\left(\omega_{\lambda}-\omega_{0}\right) t}-\sum_{\lambda} g_{\lambda}^{*} g_{\lambda} \int_{0}^{t} C_{0}^{\mathrm{e}}\left(t^{\prime}\right) \mathrm{e}^{-\mathrm{i}\left(\omega_{\lambda}-\omega_{0}\right)\left(t-t^{\prime}\right)} \mathrm{d} t^{\prime} .
$$


Equation (6) is of central importance in this work: solving this equation of motion provides the time-dependent excitation amplitude of the single emitter, hence quantifying the efficiency of single-photon absorption. The right-hand side (rhs) of equation (6) consists of two terms. The first term accounts for excitation of the atom by the single-photon wavepacket. The second term accounts for de-excitation of the two-level system by emission of a photon. Two facts are immediately obvious: firstly, equation (6) reduces to the Weisskopf-Wigner theory of SE when the atom is excited and all the modes of the field are empty initially [50]. Secondly, the maximum excitation probability of the atom depends on the shape and duration of the incident photon wavepacket. Indeed, the incident photon wavepacket is the only channel for driving the atom, while any excitation is continuously subject to exponential decay due to vacuum fluctuations. In the Weisskopf-Wigner approximation, the second term of the rhs in equation (6) is memoryless, and can be simplified to $\Gamma C_{0}^{\mathrm{e}}(t) / 2$, where $\Gamma=2 \pi \sum_{\lambda} g_{\lambda}^{*} g_{\lambda} \delta\left(\omega_{\lambda}-\omega_{0}\right)$ is the total SE decay rate. By decoupling $C_{0}^{\mathrm{e}}(t)$ from the time dependence of $C_{\lambda}^{\mathrm{g}}(t)$, namely by combining the two first-order differential equations defined by equation (3) into a second-order differential equation defined by equation (6), one only needs to know $C_{\lambda}^{\mathrm{g}}(0)$ in order to solve for $C_{0}^{\mathrm{e}}(t)$. In equation (6), $C_{\lambda}^{\mathrm{g}}(0)$ represents the mode distribution in the incident single-photon wavepacket. The entire spatial structure of the photonic modes surrounding the emitter is implicitly encoded in the coupling strength $g_{\lambda}$, which can be further expressed as

$$
g_{\lambda}=\frac{\boldsymbol{\mu} \cdot \boldsymbol{E}_{\lambda, 1}(\boldsymbol{r}=0)}{\hbar},
$$

where $\boldsymbol{\mu}$ is the dipole moment of the emitter and $\boldsymbol{E}_{\lambda, 1}(\boldsymbol{r}=0)$ is the normalized single-photon field strength for mode $\lambda$ at the position $\boldsymbol{r}=0$ of the emitter.

In the above, we have assumed that the fields belong to a finite quantization volume. In a $1 \mathrm{D}$ or quasi-1D waveguide system, a continuous-mode quantization scheme needs to be adopted. We assume that the single-photon wavepacket supported by the $1 \mathrm{D}$ waveguide can be decomposed into cylindrical waves, i.e. $\boldsymbol{E}_{\lambda}(\boldsymbol{r})=\boldsymbol{E}_{\lambda}(x, y) \mathrm{e}^{\mathrm{i}(\beta z-\omega t)}$, with the mode label $\lambda=\{m, p, \beta, q\}$, where $\beta$ is the component of the wave vector along the $z$-axis, $q$ represents the magnitude of the wavevector perpendicular to the $z$-axis, $m$ is the angular momentum and the index $p$ is used to distinguish between two degenerate polarization modes for given $m, \beta$ and $q$. For further details of the normalization of these continuous modes and the substitution of summations over discrete modes by integration over continuous modes, see appendix A. We also assume that the incoming single-photon wavepacket has a very narrow bandwidth, of the order of $10^{9} \mathrm{rad} \mathrm{s}^{-1}$, since its temporal duration is comparable to the SE lifetime. Such a narrow bandwidth with respect to the atomic transition frequency $\omega_{0}=10^{15} \mathrm{rad} \mathrm{s}^{-1}$ ensures the validity of the linear dispersion relation, i.e. $\beta_{\omega}-\beta_{0}=\left(\omega-\omega_{0}\right) / v_{\mathrm{g}}$, where $v_{\mathrm{g}}$ is the group velocity of the propagating mode. From equation (A.1) and the Weisskopf-Wigner approximation, one obtains

$\frac{\mathrm{d} C_{0}^{\mathrm{e}}(t)}{\mathrm{d} t}=-\frac{\mathrm{i} \mathrm{e}^{\mathrm{i} \omega_{0} t}}{\sqrt{v_{\mathrm{g}}} \hbar} \boldsymbol{\mu} \cdot\left[\int \mathrm{d} \omega \sum_{\kappa} \chi_{\omega, \kappa}^{g}(0) \boldsymbol{E}_{\omega, \kappa, 1, \mathrm{con}}(\boldsymbol{r}=0) \mathrm{e}^{-\mathrm{i} \omega t}\right]-\Gamma C_{0}^{\mathrm{e}}(t) / 2$

where $\chi_{\omega, \kappa}^{g}(t)=\frac{C_{\omega, \kappa}^{g}(t)}{\sqrt{\triangle \beta v_{\mathrm{g}}}}$, and $\kappa$ is given by $\kappa=\{m, p, q\}$. Equation (7) can be used to calculate the excitation probability of the single atom as illuminated by a single-photon wavepacket in a complex photonic environment, e.g. in a photonic cavity or near an optical antenna. 


\subsection{Dynamics of absorption, reflection and transmission in one-dimensional (1D) and quasi-1D waveguides}

In this section, we consider the dynamic response of both the emitter and the photon field, taking as the initial condition a single-photon wavepacket incident from the $-z$-direction, traveling towards the emitter at $z=0$. The transverse distribution of the waveguide mode is independent of $z$, and the longitudinal wave number of the modes $k$ is assumed to obey the linear dispersion relation $k-k_{0}=\left(\omega-\omega_{0}\right) / v_{\mathrm{g}}$ in the frequency range of interest. In particular, in the remainder of the paper we consider waveguides that are small enough to support only a single mode at the frequency of interest. Furthermore, we focus on nanophotonic waveguides that capture most of the emission of the nearby emitter into this waveguide mode. Thus, while our work so far was valid generally, we expect that these requirements imply that all practical realizations of predictions in the remainder of the paper require nanophotonic waveguides. Indeed, while micron-sized waveguides may still be single mode, the requirement of having a significant waveguide Purcell factor immediately translates into the requirement that the waveguide has a subwavelength mode area. With current technology this is only believed to be achievable by using high-index contrast nanowires, photonic crystals or plasmon wires. The dependent variable time $t$ has a one-to-one correspondence to the position of the peak of the singlephoton wavepacket. Initially, the peak of the pulse is at the position $z=-Z_{0}$, and the interaction between the single-photon wavepacket and the emitter reaches its maximum at $t=Z_{0} / v_{\mathrm{g}}$. At approximately this time, the emitter reaches its maximum excitation probability. Meanwhile, the emitter also loses atomic excitation probability due to the fact that it decays via SE in both the forward and backward directions. The emitted light can interfere with the incident beam, which results in extinction and pulse reshaping in the transmitted channel. The relevant timescale for de-excitation by SE is the lifetime $1 / \Gamma$, where $\Gamma$ is the SE decay rate of the emitter. The singlephoton wavepacket will be redistributed into a (re-shaped) reflected and a transmitted pulse and the emitter will relax to the ground state in several lifetimes after attaining the maximum excitation probability.

In any $1 \mathrm{D}$ waveguide, the continuous-mode variable can equivalently be taken as the wavevector $k$ or the frequency $\omega_{k}$ due to the linear dispersion approximation. The wavevector $k$ is chosen here. In such an infinitely long single-mode waveguide, the SE divides equally between the forward and backward propagating channels. If we assume that the incident photon wavepacket is a single packet offered from just one direction, this means that the SE $\beta_{0}$-factor for the pumping channel is at most $50 \%$. For any given incident photon wavepacket $C_{k}^{g}(0)$, it is straightforward to calculate the dynamic atomic excitation $C_{0}^{\mathrm{e}}(t)$ in the $1 \mathrm{D}$ waveguide, which is given by

$$
\frac{\mathrm{d} C_{0}^{\mathrm{e}}(t)}{\mathrm{d} t}=-\mathrm{i} \int_{-\infty}^{\infty} \mathrm{d} k C_{k}^{g}(0) g_{k} \mathrm{e}^{-\mathrm{i}\left(\omega_{k}-\omega_{0}\right) t}-\Gamma C_{0}^{\mathrm{e}}(t) / 2
$$

In our case, the coupling strength $g_{k}$ is assumed to be frequency-independent due to the narrow bandwidth of the input single-photon pulse and is given as $g_{k}=\sqrt{\frac{\Gamma}{4 \pi} v_{\mathrm{g}}}$ [22].

Once the atomic excitation $C_{0}^{e}(t)$ is known, one can calculate the dynamics of $C_{k}^{g}(t)$ to obtain the reflected and transmitted wavepackets. According to equation (5), the total probability amplitude of the forward propagating wavepacket is a coherent superposition of the initial 
incident photon wavepacket and the emitted photon and is given by

$$
C_{k,+}^{g}(t)=C_{k}^{g}(0)-\mathrm{i} \int_{0}^{t} C_{0}^{\mathrm{e}}\left(t^{\prime}\right) g_{k}^{*} \mathrm{e}^{\mathrm{i}\left(\omega_{k}-\omega_{0}\right) t^{\prime}} \mathrm{d} t^{\prime} .
$$

For the backward direction, there is no incident term $\left(C_{k,-}^{g}(0)=0\right)$. Hence, the corresponding probability amplitude is

$$
C_{k,-}^{g}(t)=-\mathrm{i} \int_{0}^{t} C_{0}^{\mathrm{e}}\left(t^{\prime}\right) g_{k}^{*} \mathrm{e}^{\mathrm{i}\left(\omega_{k}-\omega_{0}\right) t^{\prime}} \mathrm{d} t^{\prime}
$$

In most realistic cases, an emitter coupled to a $1 \mathrm{D}$ waveguide will still have a residual coupling to free space modes that are not guided by the wire. For such a quasi-1D waveguide, the SE $\beta$-factor into the forward plus backward propagating waveguide modes will have a value less than $100 \%$. Also, a break in symmetry in the geometry might imply that emission into forward and backward propagating waveguide modes is unbalanced. In these cases where the pumping channel funneling the incident single-photon wavepacket has an SE $\beta$-factor of $\beta_{0}$, equation (8) becomes

$$
\frac{\mathrm{d} C_{0}^{\mathrm{e}}(t)}{\mathrm{d} t}=-\mathrm{i} \int_{-\infty}^{\infty} \mathrm{d} k C_{k}^{g}(0) \sqrt{\frac{\beta_{0} \Gamma}{2 \pi} v_{\mathrm{g}}} \mathrm{e}^{-\mathrm{i}\left(\omega_{k}-\omega_{0}\right) t}-\Gamma C_{0}^{\mathrm{e}}(t) / 2 .
$$

It should be remarked that the first term of the rhs in equation (11) is simply the pulse shape in the absence of light-matter coupling, assuming that the coupling strength $g_{k}$ is a constant. In the next section, we will study specific wavepackets and make use of the equation of motion derived here.

\section{Gaussian pulse excitation of a 1D waveguide}

First we consider Gaussian input single-photon wavepackets, defined as $C_{k}^{g}(0)=$ $\left[\frac{2}{\pi \Delta^{2}}\right]^{\frac{1}{4}} \mathrm{e}^{\left[\mathrm{i}\left(k-k_{0}\right) Z_{0}-\frac{\left(k_{0}-k\right)^{2}}{\Delta^{2}}\right]}$, where $-Z_{0}$ is the position at which the peak of the pulse passes at $t=0$. The relation $\int_{\infty}^{\infty}\left|C_{k}^{g}(t=0)\right|^{2} \mathrm{~d} k=1$ ensures that there is only a single photon in the wavepacket. With the linear dispersion relation and the substitutions of $t_{0}=\frac{Z_{0}}{v_{\mathrm{g}}}$ and $\Omega=\Delta v_{\mathrm{g}}$, one can obtain an analytical solution for the dynamics of single-photon absorption, i.e. for the excitation amplitude of the two-level system

$$
C_{0}^{\mathrm{e}}(t)=s \frac{1}{2} \sqrt{\frac{\pi}{a}} \mathrm{e}^{\frac{b^{2}}{a}-c}\left[\operatorname{erf}\left(\sqrt{a} t+\frac{b}{\sqrt{a}}\right)-\operatorname{erf}\left(\frac{b}{\sqrt{a}}\right)\right] \mathrm{e}^{-\frac{\Gamma}{2} t},
$$

where $s=-\mathrm{i} \sqrt{\frac{\Gamma}{4 \pi}}\left[2 \pi \Omega^{2}\right]^{\frac{1}{4}}, a=\frac{\Omega^{2}}{4}, b=-\left(\Gamma-\Omega^{2} t_{0}\right) / 4, c=\Omega^{2} t_{0}^{2} / 4$, and $\operatorname{erf}(x)$ is the error function, defined as $\operatorname{erf}(x)=\frac{2}{\sqrt{\pi}} \int_{0}^{x} \mathrm{e}^{-t^{2}} \mathrm{~d} t$. The result $C_{0}^{\mathrm{e}}(t)$ obtained from the time-dependent theory $[46,49]$ explicitly describes the atomic excitation as a function of time. This highly important information is not contained in the purely stationary results of [22, 39], since in purely stationary theory the infinite extent of the incident package directly implies identically zero atomic excitation. As shown in [44], stationary theory [22, 39] can be extended to obtain the time-dependent $C_{0}^{\mathrm{e}}(t)$ via the calculation of the stationary interacting eigenstates, projection of the initial state and application of the time evolution operator. Since our formalism does not require eigenstates to be calculated, we anticipate that it will be most easily generalized to also deal with multimode or multiple scattering systems. We would like to point out that a third 


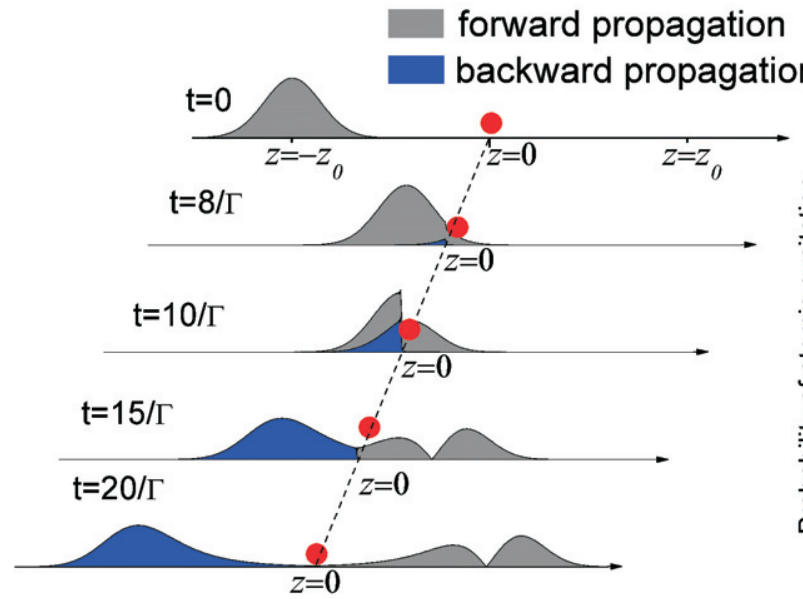

(a)

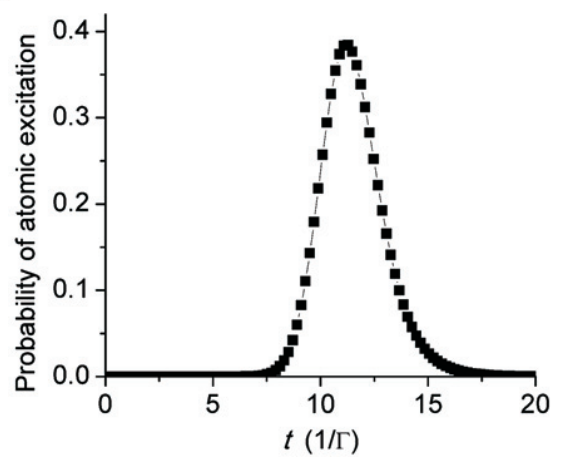

(b)

Figure 2. (a) Snapshots of the amplitudes of the transmitted and reflected wavepackets as distributed along the spatial axis at different instances of time; (b) the time dependence of the atomic excitation. At $t=0$, the peak of the Gaussian input single-photon wavepacket is localized at $z=-Z_{0}=-10 v_{\mathrm{g}} / \Gamma$, with FWHM equal to $\Gamma$. The red dot indicates the fixed position of the emitter.

alternative route to obtain $C_{0}^{\mathrm{e}}(t)$, that likewise includes the initial condition and does not require eigenstates to be calculated first, is the Laplace transform method developed by one of us (see Wubs et al [52]). We found that the two methods are equivalent and yield the same result for $\left|C_{0}^{\mathrm{e}}(t)\right|^{2}$. The derivation of $\left|C_{0}^{\mathrm{e}}(t)\right|^{2}$ based on the Laplace transform method is briefly outlined in appendix A.

Figure 2(a) shows the spatial variations of the forward- and backward-propagating wavepackets, which are obtained by Fourier transforming $C_{k,+}^{g}(t)$ and $C_{k,-}^{g}(t)$ with respect to $k$. Initially the incident single-photon wavepacket is far away $\left(z<-Z_{0}\right)$ from the emitter. At this instance there is negligible interaction. As the leading edge of the pulse reaches the emitter, the interaction is effectively switched on. From this time onwards, the emitter gains excitation amplitude, and starts to emit an outgoing wavepacket into both the forward and backward directions. Before the emitter reaches its maximum excitation shown in figure 2(b), one notices that the forward-propagating wavepacket experiences a sharp drop at the position of the emitter, while the backward-propagating wavepacket experiences a steady increase of its magnitude at $z=0$. Figure 2(b) shows the time dependence of the corresponding atomic excitation, which will be discussed in section 3.2. The sharp drop in the forward-propagating wavepacket is due to the transfer of energy from light to the emitter, the rate of which is larger than the SE decay rate of the emitter itself, given our assumption on the coupling strength $g_{k}$, set by the mode profile and $v_{\mathrm{g}}$. In contrast to the forward-propagating packet, the backward-propagating wavepacket obtained purely from emission has an increasing magnitude. After reaching the maximum excitation, the relaxation of the emitter leads to decreasing magnitudes for both the forwardand backward-propagating wavepackets. Moreover, the forward-propagating wavepacket is seen to be strongly reshaped due to the interference of the incident packet with the emitted light. The resulting minimum in the forward-propagating wavepacket will be further discussed in the stationary limit in section 3.1. One also notes that the trailing edges of the two wavepackets are 
longer than the leading edges. The widths of the leading edges are determined by the incoming single-photon wavepacket, while the trailing tails are essentially determined by the lifetime of the excited state of the emitter.

\subsection{Stationary limits of the single-photon absorption in a $1 D$ waveguide}

We now examine our theory in the stationary limit, to connect our dynamic results to earlier work by Shen and Fan [39]. In our formalism, the stationary limit essentially corresponds to choosing the incident single-photon pulses to have temporal width tending to infinity, implying a concomitantly low instantaneous incident amplitude and hence that the emitter remains essentially in the ground state at all times. From our dynamic theory, we can extract transmission and reflection amplitudes in the limit of long excitation pulses, and at times far later than the time window in which interaction with the atom has taken place. Using a Fourier transform, we extract frequency-dependent reflection and transmission amplitudes. We compare these frequency-dependent coefficients, which we refer to as the stationary limit of our dynamic theory, with the frequency-dependent coefficients obtained in purely stationary theory by Shen and Fan [39]. In the stationary limit, in which the emitter has relaxed into its ground state, the forward- and backward-propagating wavepackets are essentially the transmitted and reflected light, similar to light scattering by an impurity in a $1 \mathrm{D}$ waveguide. If we take a snapshot long after the interaction time (we use $t_{\mathrm{s}}=100 / \Gamma$, but our results are independent of this choice), we find instantaneous reflection and transmission amplitudes in $k$-space. By taking advantage of the linear dispersion relation, we can convert these $k$-space instantaneous amplitudes into spectral information. The transmission and reflection spectra can be extracted as

$$
\begin{aligned}
& T(\omega)=\left|C_{\omega_{k},+}^{\mathrm{g}}\left(t_{\mathrm{s}}\right) / C_{\omega_{k}}^{\mathrm{g}}(0)\right|^{2}, \\
& R(\omega)=\left|C_{\omega_{k},-}^{g}\left(t_{\mathrm{s}}\right) / C_{\omega_{k}}^{\mathrm{g}}(0)\right|^{2} .
\end{aligned}
$$

At first sight, the coexistence of the time and frequency dependence in the transmission and reflection spectra may appear odd. However, for times $t_{\mathrm{s}}$ much later than the interaction time, the time $t_{\mathrm{s}}$ is essentially equivalent to a spatial variable $z=v_{\mathrm{g}} t_{\mathrm{s}}$. Hence, the transmission and reflection spectra can be interpreted as frequency signals monitored at a certain position.

Figure 3 shows the spectral intensities of the transmitted wavepacket and the reflected wavepacket, as well as the transmission and reflection spectra in the stationary limit, at $t_{\mathrm{s}}$, i.e. long after the interaction of the emitter with the incident wavepacket. Figures 3(a) and (b) show that the spectral intensity of transmitted light has a dip at the resonance frequency, while the spectral intensity of the reflected light resembles the original pulse shape. The dip corresponds to the minimum in the forward-propagating wavepacket in figure 2(a) due to the resonant interaction with the emitter, and the dip magnitude depends on the bandwidth of the incident wavepacket. We also note that narrower bandwidth will yield more reflection of light. Figure 3(c) shows the transmission and reflection spectra in the stationary limit, by normalizing the reflected and transmitted pulse spectra to the spectrum of the incident wavepacket. We now compare the transmission and reflection spectra from our limiting procedure with stationary solutions obtained by solving for the eigenstates of the system as reported by Shen and Fan [39]. Such stationary behavior of the transmission and reflection can be well modeled by solving for the eigenstates of the system. These eigenstates can also be used as a complete basis for 

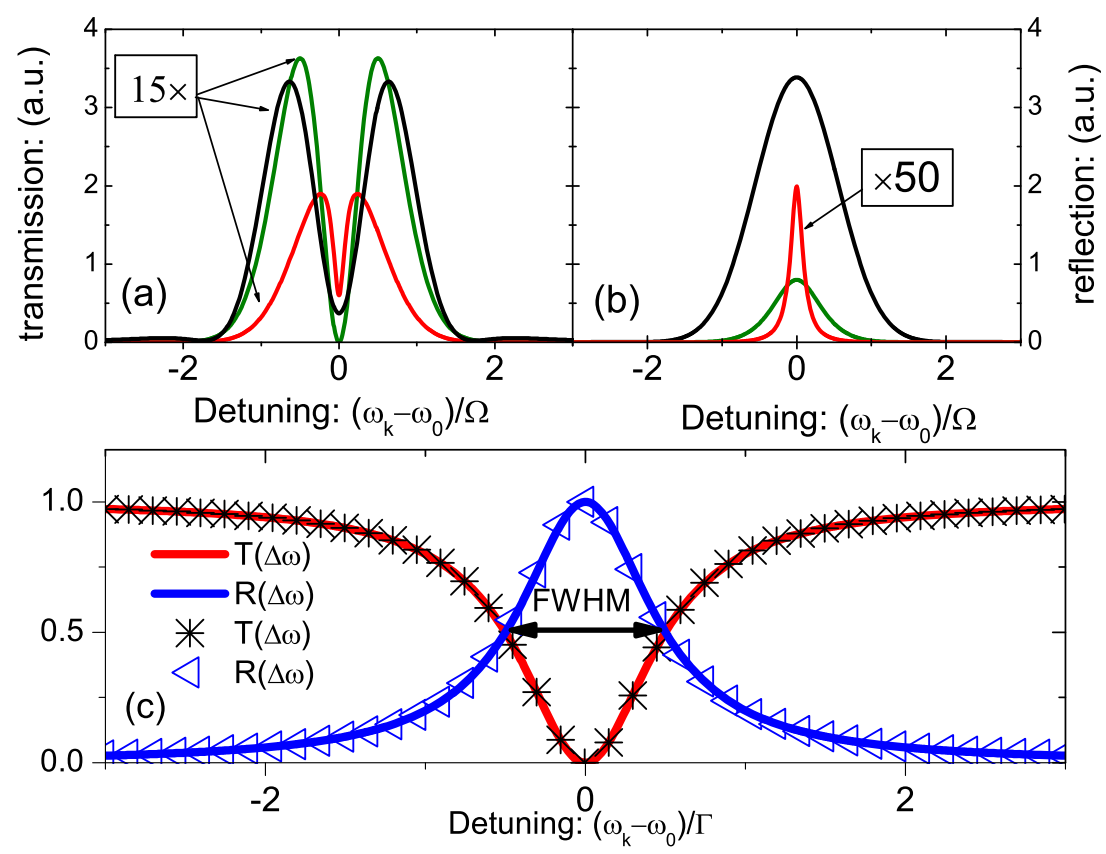

Figure 3. (a) Transmitted spectral intensity and (b) reflected spectral intensity of a Gaussian incident single-photon wavepacket mediated by a single emitter. The plotted results are for different ratios of pulse bandwidth $\Omega$ to decay rate $\Gamma$. Note that the $x$-axis represents frequency in units of the pulse bandwidth in (a) and (b), but in units of decay rate in (c). In (a) and (b), we find that as the ratio of bandwidth to decay rate is decreased from large $(5 \Gamma$, red) to small $(0.2 \Gamma$, black), the reflected intensity appears to increase strongly. The transmitted intensity shows a pronounced minimum at zero detuning. This minimum is deepest, reaching $T=0$, when the incident pulse width exactly matches the lifetime of the emitter (dark green, $\Omega=\Gamma$ ). Panel (c) shows the transmission and reflection spectra, obtained by normalizing the transmitted and reflected intensity as plotted in (a) and (b) to the intensity spectrum of the incident wavepacket. The stars and triangles correspond to the results extracted from our time-dependent formalism, via equation (12). The solid lines show the stationary calculations.

time evolution, as reported by Rephaeli and Shen [44]. For a given detuning $\delta=\omega_{k}-\omega_{0}$, the corresponding reflection and transmission spectra can be described as follows [39]:

$$
\begin{aligned}
& T(\delta)=\left|\frac{\delta}{\mathrm{i} \Gamma / 2+\delta}\right|^{2}, \\
& R(\delta)=\left|-\frac{\mathrm{i} \Gamma / 2}{\mathrm{i} \Gamma / 2+\delta}\right|^{2} .
\end{aligned}
$$

The transmission and reflection spectra that our time-dependent theory predicts using a Gaussian input wavepacket with a narrow bandwidth agree well with Shen's stationary model defined by equation (15), as shown in figure 3(c). We would also like to point out that the transmission and reflection spectra for a narrow bandwidth pulse in our stationary limit are independent of $\Omega$. This independence, which is rigorous only in the limit of zero bandwidth, is confirmed by the 
identical transmission and reflection spectra for the three pulses in figures 3(a) and (b). Based on the stationary model, the full-width that half-maximum (FWHM) of transmission and reflection equals the total decay rate of the emitter, i.e. $\mathrm{FWHM}=\Gamma$.

\subsection{Time dependence of the transmittance and reflectance of the single-photon wavepacket in a $1 D$ waveguide}

The stationary model accounts for the transmission and reflection at times long after the interaction for any given frequency distribution. Indeed, a good approximation of the transmitted and reflected wavepackets is obtained simply by multiplication of the incident spectrum, with transmission and reflection coefficients combined with the right phase factor. However, the calculated transmission and reflection coefficients in the stationary limit reported in figure 3 and [39] by itself provide no means to assess the time evolution of the atomic excitation during the photon emitter interaction. This dynamic information is an essential result of our work that we now proceed to discuss. Also, the model allows one to obtain the time-dependent transmission and reflection probabilities at the time of interaction, instead of being limited to times much later than the interaction interval. We define the time-dependent transmission (reflection) probabilities by including all amplitude traveling to the right (left) as follows:

$$
\begin{aligned}
& \mathrm{T}(t)=\int_{-\infty}^{\infty} \mathrm{d} k\left|C_{k,+}^{\mathrm{g}}(t)\right|^{2}, \\
& \mathrm{R}(t)=\int_{-\infty}^{\infty} \mathrm{d} k\left|C_{k,-}^{\mathrm{g}}(t)\right|^{2} .
\end{aligned}
$$

It is important to realize here that these coefficients are defined not via spatially separating the amplitude present to the left/right of the atom, but strictly by separating the forward and backward directions by wave vector. As opposed to the reflection and transmission coefficients in the stationary limit, these instantaneous coefficients have no frequency content. Instead, they allow us to monitor the distribution of excitation between the atom and the forward and backward emission channels as a function of time. The frequency-dependent transmission and reflection reported in figure 3 can be obtained by evaluating $T(t)$ and $R(t)$ for large times (e.g. $t>100 / \Gamma$ ), for many narrow-band initial conditions centered at different frequencies.

In figure 4, we consider the time-dependent probabilities for the atomic excitation, transmission and reflection. For an incoming single-photon wavepacket with FWHM equal to $\Gamma$, we found that the atomic excitation increases as a function of time as the peak of the pulse approaches. After $t=10 / \Gamma$, the atomic excitation decays with a time constant give by the lifetime of the emitter $(1 / \Gamma)$, after achieving a maximum value of atomic excitation close to $40 \%$, see figure 4(a). The reflection builds up with time due to the continuous re-emission of light from the emitter. In contrast, the forward packet has a nonmonotonic, although generally decreasing behavior. The kink in $T(t)$ is due to the interference of the incident packet and re-emitted light. In contrast to the prediction of the stationary model, the transmitted wavepacket does not vanish, although the incident wavepacket is tuned exactly to resonance. The imperfect reflection as compared to the perfect reflection in the stationary limit is due to the fact that optimum atomic excitation requires a finite pulse length, or equivalently finite bandwidth of the incident packet, whereas perfect reflection only occurs at a single frequency. The impact of the finite bandwidth of the incoming wavepacket on the maximum value of the atomic excitation is also evident in figure 4(b), which shows the atomic excitation probability as a function of 

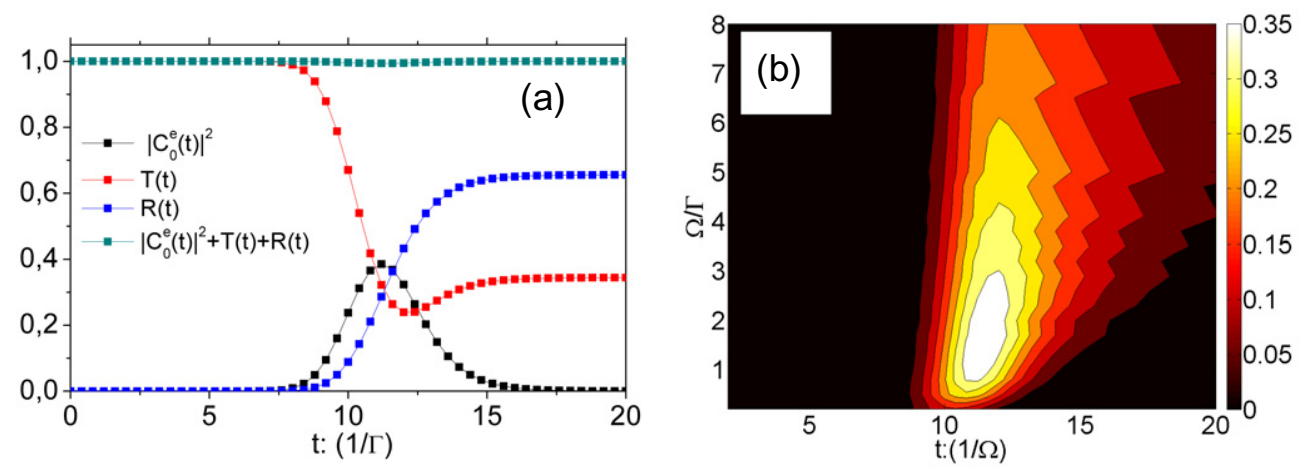

Figure 4. (a) Time dependence of the transmission and reflection probability of the incident single-photon wavepacket and the atomic excitation. (b) Contour plot of the atomic excitation probability versus time and bandwidth of the incoming Gaussian wavepacket.

time for different bandwidths of the incoming wavepacket. The graph clearly shows that there is a range of optimum bandwidths that lead to atomic excitation probability with a maximum value of around $40 \%$. This range of optimum bandwidths is comparable to $\Gamma$, corresponding to incident photon wavepackets that have a duration comparable to the SE decay time. The $40 \%$ atomic excitation is surprisingly high given that reciprocity sets the fundamental limit for excitation from just one direction in the waveguide to $50 \%$. The value of $40 \%$ could be pushed closer to the limit of $50 \%$ by not using Gaussian pulses, but rather the inverting pulses proposed by Stobińska et al [11, 23, 43, 44]. However, exceeding the limit of 50\% will invariably require illuminating the emitter from two sides with a proper phase relation between the two input pulses. In the following, we show that instead of increasing the complexity of illumination by using two pulses, it is also possible to further increase the excitation probability above $50 \%$ by engineering the photonic environment of the emitter to have broken symmetry.

\section{Gaussian input on a semi-infinite 1D waveguide}

For an infinitely long 1D waveguide, the atomic excitation probability is prevented from reaching unity by the fact that the emitter can decay equally into two directions, namely the forward and backward directions. Essentially, the coupling efficiency of the emitter with the one-sided pumping channel, or conversely the SE $\beta$-factor in just one waveguide direction, is at best $50 \%$. In order to obtain higher atomic excitation probability, all the channels into which the emitter decays should be suppressed, except for the one optical input/output channel through which the incident photon wavepacket is sent in. In this section, we consider a situation where the single-photon wavepacket is launched into a semi-infinite 1D waveguide. By placing the emitter at an optimized distance from the waveguide termination, we expect that the coupling of the emitter to the input waveguide mode is optimized.

\subsection{Enhanced spontaneous emission (SE) and the SE $\beta$-factor by a terminated metallic nanowire}

Specifically, we study a terminated metallic nanowire coupled to an emitter, as shown in figure 5(a). On the right, the metallic nanowire is terminated with a spherical endcap of radius 

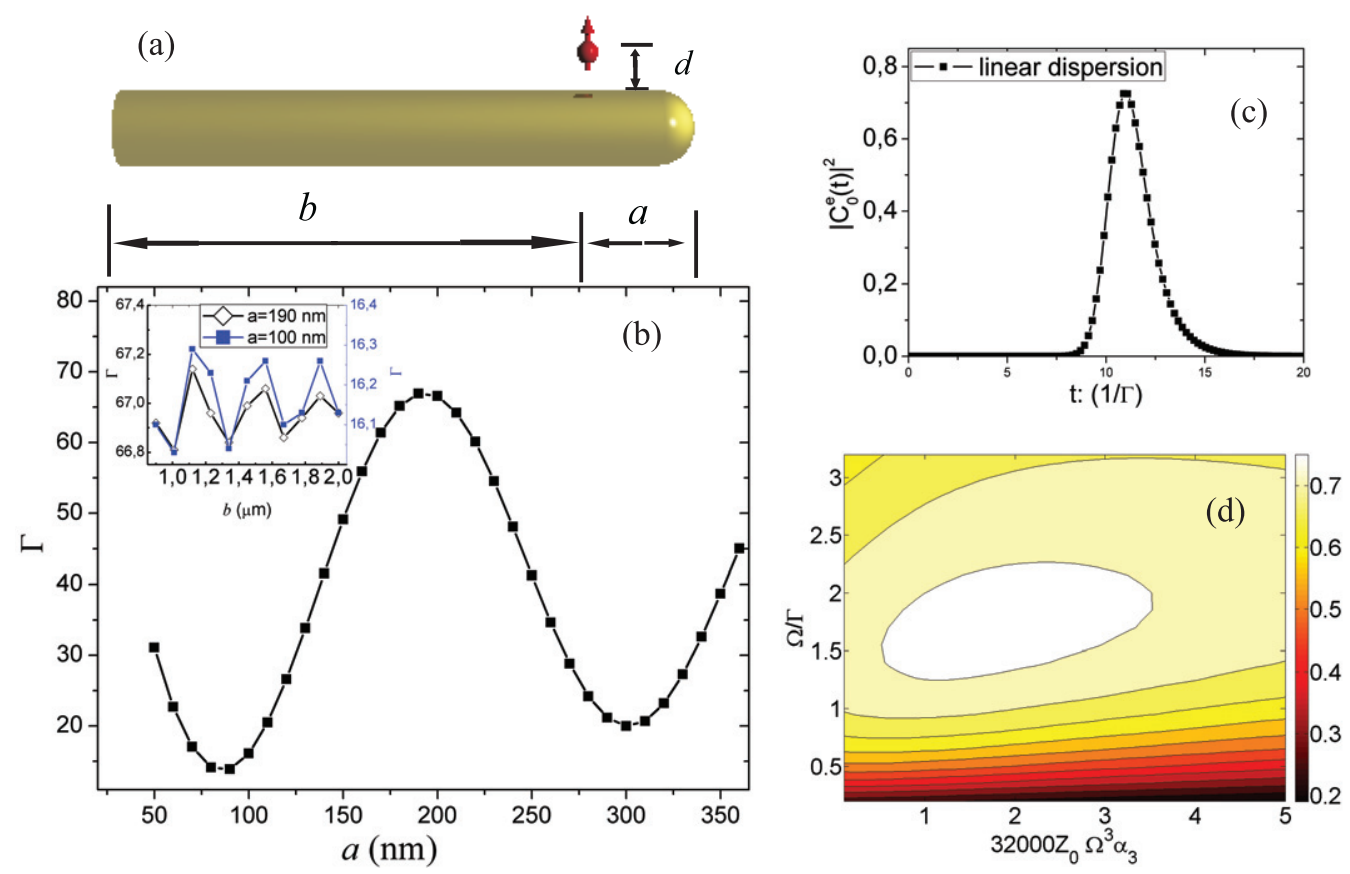

Figure 5. (a) Sketch of the terminated metallic nanowire coupled to a sine emitter. (b) Position dependence of the total decay rate of the emitter coupled to the half truncated metallic nanowire. Here $a$ denotes the distance of the emitter to the rod end on the right. The $20 \mathrm{~nm}$ radius silver nanowire $\left(\varepsilon_{A g}=-50+0.6 j\right)$ is embedded in the host material with index $n=1.414$. The inset in (b) shows the total decay rate as a function of the length of the modeling domain, which does not give any significant impact on $\Gamma$; for further details, see [38]. (c) Time dependence of the atomic excitation for a terminated metallic nanowire with an SE $\beta$-factor of $91 \%$. (d) Contour plot of the maximum of the atomic excitation versus bandwidth and $\alpha_{3}$ for a Gaussian input wavepacket in a terminated nanowire with $\alpha_{2}=0$.

$R$ (equal to the radius of the nanowire). On the left, the nanowire is infinitely long. As shown in figure 5(a), $a$ is the distance of the emitter to the right rod end, and $d$ is the distance to the wire edge. Figure 5(b) shows the variation of the decay rate as a function of $a$ for $d=10 \mathrm{~nm}$ and an emission wavelength of $1000 \mathrm{~nm}$. We obtained numerical results using finite-element method (FEM) calculations, as explained in our previous work [38]. Such FEM modeling is rather flexible and can handle complex photonic structures [54] compatible with current lithographic fabrication technology. For an infinitely long $20 \mathrm{~nm}$ radius silver nanowire, there are only two guided plasmonic modes, i.e. a forward and a backward propagating guided mode with propagation constants $\pm k$ and corresponding wavelength $\lambda_{\text {eff }}=2 \pi / k=436 \mathrm{~nm}$. The decay rate into the forward- and backward-propagating plasmon modes is 33.8 when normalized to the decay rate of the emitter in vacuum. Accordingly, the decay rate into one direction is $\gamma_{\mathrm{pl}, 0} \sim 17$. The total decay rate coupled to an infinitely long nanowire, including the decay into radiative modes in the continuum and direct quenching, is $\Gamma=38.27$. Hence, the $\beta$-factor into the pumping channel is approximately $44 \%$. For a terminated metallic nanowire, the total 
decay rate shows a clear interference pattern as a function of the distance of the emitter to the termination. This pattern is exactly the 1D analogue of Drexhage's observation that the lifetime of a molecule in front of a mirror oscillates as a function of the mirror-emitter separation [53]. Compared to the infinitely long wire with $\Gamma=38.27$, we can enhance or inhibit $\Gamma$ by a large amount, up to $\sim 80 \%$, by tuning the source-termination distance $a$. Importantly, by integrating the power flux in the transverse plane of the plasmon nanowire for $a=190 \mathrm{~nm}$, we found that $91 \%$ of the SE is coupled into the guided mode that exits the plasmon wire on the left. We thus also expect up to $91 \%$ excitation efficiency when offering a single-photon wavepacket through the waveguide.

The mechanism responsible for the enhancement is that the waveguide termination essentially functions as a mirror, which reflects the right propagating plasmonic mode to the left. Similar to image dipole theory for Drexhage's experiment, which predicts a twofold enhancement of the SE decay rate for a dipole emitter in front of and perpendicular to a mirror, the waveguide termination also acts as a mirror, which yields an enhancement factor of 2 of the SE into the plasmonic modes. Consequently, the emission rate into just the left propagating plasmonic mode is approximately enhanced by a factor of 4 since the twofold enhancement is distributed over just half the channels. Compared to the infinitely long nanowire, such termination with a proper distance to the emitter gives rise to a SE $\beta$-factor up to $91 \%$ for the pump channel.

\subsection{Single-photon wavepacket propagation along the terminated metallic nanowire}

Having quantified the SE decay rate enhancement at the wire termination using FEM modeling, we now proceed to model the probability of absorption of a single-photon wavepacket. To this end we first need to estimate the shape of the wavepacket including the reflected part, for which we need to know the complex reflection coefficient at the wire end. We can estimate the complex reflection coefficient from the calculated decay rate. Indeed, if the reflection coefficient at the rod end is $r \mathrm{e}^{\mathrm{i} \theta}$, the decay rate of the emitter influenced by the rod end can be approximated as $\frac{\gamma_{\mathrm{pl}}}{\gamma_{\mathrm{pl}, 0}} \propto\left|E_{\mathrm{t}}\right|^{2} /\left|E_{0}\right|^{2}=\left|E_{0}\left(1+r \mathrm{e}^{\mathrm{i} \theta} \mathrm{e}^{\mathrm{i} 2 k_{0} a}\right)\right|^{2} /\left|E_{0}\right|^{2}$, where $E_{0}$ is the electric field for the plasmonic mode without reflections, $E_{\mathrm{t}}$ is the total field including the left-hand propagating plasmonic mode and the one reflected from the right. From figure 5(b), we extract the reflection parameter $\left|1+r \mathrm{e}^{\mathrm{i} \theta} \mathrm{e}^{\mathrm{i} 2 k_{0} a}\right| \sim 1.9$, since $\frac{\gamma_{\mathrm{pl}}}{\gamma_{\mathrm{pl}, 0}} \sim 3.6$ according to our FEM calculations.

We now use the reflection coefficient to construct the full wavepacket driving the atom, assuming that we initially launch a Gaussian wavepacket $\phi_{0}(z, t)=$ $\left(2 \pi \Delta^{2}\right)^{1 / 4} \mathrm{e}^{\left[\mathrm{i}\left(k_{0} z-\omega_{0} t\right)\right]} \mathrm{e}^{\left[-\frac{\Delta^{2}\left(z-v_{\mathrm{g}} t+Z_{0}\right)^{2}}{4}\right]}$ from $z=-Z_{0}$ at $t=0$ into the waveguide. The backwardpropagating wavepacket due to the reflection can be written as

$$
\phi_{r}(Z, t)=r \mathrm{e}^{\mathrm{i} \theta}\left(2 \pi \Delta^{2}\right)^{1 / 4} \mathrm{e}^{\mathrm{i} k_{0} a} \mathrm{e}^{\left(\mathrm{i}\left(k_{0} Z+\omega_{0} t\right)\right)} \mathrm{e}^{\left[-\frac{\Delta^{2}\left(a-v_{\mathrm{g} t}+Z_{0}-Z\right)^{2}}{4}\right]},
$$

where $Z=z-a$. Hence the total field given by $\phi_{t}(z, t)=\left[\phi_{0}(z, t)+\phi_{r}(Z, t)\right]$ at $t=0$ is

$$
\begin{aligned}
\phi_{t}(z, 0)=\left(2 \pi \Delta^{2}\right)^{1 / 4} \mathrm{e}^{\left(\mathrm{i}\left(-\omega_{0} t\right)\right)} \mathrm{e}^{\left[-\frac{\Delta^{2}\left(z+Z_{0}\right)^{2}}{4}\right]} & \\
+ & r \mathrm{e}^{\mathrm{i} \theta}\left(2 \pi \Delta^{2}\right)^{1 / 4} \mathrm{e}^{\mathrm{i} 2 k_{0} a} \mathrm{e}^{-\mathrm{i} \omega_{0} t} \mathrm{e}^{\left[-\frac{\Delta^{2}\left(z+2 a+Z_{0}\right)^{2}}{4}\right]} .
\end{aligned}
$$


We simplify this expression using one assumption, namely that the amplitude envelope factor $\mathrm{e}^{\left[-\frac{\Delta^{2}\left(z+2 a+Z_{0}\right)^{2}}{4}\right]}$ in factor $\mathrm{e}^{\left[-\frac{\Delta^{2}\left(z+Z_{0}\right)^{2}}{4}\right]}$ of in the second term can be simplified to be equal to the amplitude envelope single-photon wavepackets with a bandwidth of a few gigahertz corresponding to an envelope length of a few tens of centimeters. The offset $2 a$ in the envelope factor of the second term, which is $2 a=380 \mathrm{~nm}$, is negligible compared to the entire envelope length. In other words, the femtosecond travel time between the emitter and the wire termination is far below the nanosecond temporal length of the pulse envelope. As a consequence, we can take the envelope of the reflected pulse to be identical to that of the incident wavepacket. In this approximation, the total field that appears in the excitation term is

$$
\phi_{\mathrm{t}}(z, 0) \simeq \phi_{0}(z, 0)\left[1+r \mathrm{e}^{\mathrm{i}\left(\theta+2 k_{0} a\right)}\right] .
$$

Exactly as in the case of a symmetric waveguide, the dynamics of the atomic excitation in equation (6) is set by the field amplitude $\phi_{\mathrm{t}}(k, 0)$, i.e. the Fourier transform of $\phi_{\mathrm{t}}(z, 0)$, and the coupling strength $g_{k}$ for the corresponding mode. Due to the reflection from the rod end, the field is enhanced by a factor of $\left[1+r \mathrm{e}^{\mathrm{i}\left(\theta+2 k_{0} a\right)}\right]$, as is evident in equation (20). Not only the field, but also the coupling strength $g_{k}$ is affected by the presence of the wire termination, since the decay rate into the plasmonic channel, i.e. $\Gamma_{\mathrm{pl}}=\beta_{0} \Gamma=2 \pi\left[1+r \mathrm{e}^{\mathrm{i}\left(\theta+2 k_{0} a\right)}\right]^{2} g_{k}^{2} / v_{\mathrm{g}}$, again contains the reflection at the wire termination. The overall effect of the strong reflection at the wire end on the integral kernel in the excitation term is given by

$$
\begin{aligned}
C_{k}^{\mathrm{g}}(0) g_{k} & =\left[1+r \mathrm{e}^{\mathrm{i}\left(\theta+2 k_{0} a\right)}\right] \phi_{0}(k, 0) \sqrt{\beta_{0} \Gamma v_{\mathrm{g}} /\left(2 \pi\left[1+r \mathrm{e}^{\mathrm{i}\left(\theta+2 k_{0} a\right)}\right]^{2}\right)} \\
& =\phi_{0}(k, 0) \sqrt{\beta_{0} \Gamma v_{\mathrm{g}} /(2 \pi)},
\end{aligned}
$$

where $\phi_{0}(k, 0)$ is the Fourier transform of the initially incident wavepacket $\phi_{0}(z, 0)$. Equation (21) shows that the factor $\left[1+r \mathrm{e}^{\mathrm{i}\left(\theta+2 k_{0} a\right)}\right]$ cancels out in the excitation term. Therefore, the equation defined in equation (11) has properly taken the reflection coefficient as well as the reflection phase into account, except that we assume the envelope to be constant over the spatial range from the emitter to the rod end, which is a valid approximation as discussed.

\subsection{Enhanced single-photon absorption by a terminated metallic nanowire}

We calculate the time dependence of the atomic excitation probability of an atom coupled optimally to the terminated metallic nanowire for a Gaussian input photon wavepacket, as shown in figure 5(c). For such a terminated metallic nanowire, we found that the maximum of the atomic excitation probability is $72.4 \%$. The fact that the probability of atomic excitation exceeds $50 \%$ despite the fact that light is only injected into the system from one side is a direct consequence of the high SE $\beta$-factor of $91 \%$ for unidirectional emission into the open end of the waveguide. For an ideal case with $\beta$-factor of $100 \%$, we also find that the maximum atomic excitation is $\sim 80 \%$ for a Gaussian wavepacket. The fact that there is still a discrepancy between 72.4 and $91 \%$ (resp. 80 and 100\%) shows that Gaussian input wavepackets do not form the optimum temporal pulse shapes. By pulse shaping the input wavepacket it may be possible to more perfectly approach the perfect inverting pulse.

As an example of further optimization of pulse shape, we study the possibility of shaping the input wavepacket through waveguide dispersion. Considering a highly dispersive waveguide 
with dispersion relation $\alpha(\omega)=\alpha_{0}+\alpha_{1}\left(\omega_{0}\right)(\omega-\omega)+\alpha_{2}\left(\omega_{0}\right) / 2(\omega-\omega)^{2}+\alpha_{3}\left(\omega_{0}\right) / 6(\omega-\omega)^{3}$, the Gaussian wavepacket $\phi_{0}\left(-Z_{0}, t\right)=\left(2 \pi \Delta^{2}\right)^{1 / 4} \mathrm{e}^{\left[-\mathrm{i} \omega_{0} t\right]} \mathrm{e}^{\left[-\frac{\Delta^{2}\left(-v_{\mathrm{g} g} t z_{0}\right)^{2}}{4}\right]}$ initially launched at $z=$ $-Z_{0}$ will be modified due to the waveguide dispersion. When the wavepacket reaches the emitter, the corresponding pulse shape is given by [55]

$$
\begin{aligned}
\phi_{0}(0, t)=4 \sqrt{2} & (\pi)^{3 / 4} \frac{|B|^{(-1 / 3)}}{\tau} \mathrm{e}^{\left[\left(2-3 A B-6 C^{2}\right) \frac{1}{3 B^{2}}-\mathrm{i} C\left(3 A B+2 C^{2}-6\right) \frac{1}{3 B^{2}}\right]} \\
& \times A i\left[\left(1-A B-C^{2}+\mathrm{i} 2 C\right)(|B|)^{-4 / 3}\right],
\end{aligned}
$$

where $A=4\left(t-\alpha_{0} Z_{0}\right) / \tau, B=32 Z_{0} \frac{\alpha_{3}}{\tau^{3}}, C=8 Z_{0} \frac{\alpha_{2}}{\tau^{2}}, \tau=4 /\left(\Delta v_{\mathrm{g}}\right)$ and $A i(x)$ is the Airy function. By taking into account the dispersive features of the waveguide as well as the possibility of chirping the input pulse, we found that the chirping as well as $\alpha_{2}$ are simply detrimental to reaching maximum absorption, since they mainly give rise to broadening of the pulse. Interestingly, an optimized $\alpha_{3}\left(\omega_{0}\right)$ can improve the maximum of the atomic excitation by $4 \%$ compared with the nondispersive case, as shown in the contour plot of maximized atomic excitation as a function of bandwidth $(\Omega)$ and $\alpha_{3}$ in figure 5(d). In closing, we have shown that a considerable amount of atomic excitation can be gained simply by operating at the termination of the plasmonic waveguide.

\section{Conclusion}

In conclusion, we have theoretically analyzed the time dependence of single-photon absorption by a single emitter. The model allows us to specify any single-photon wavepacket as the initial condition and to calculate the time dependence of the atomic excitation. We apply the theoretical model to quasi-1D waveguides coupled to a single emitter. For a Gaussian singlephoton wavepacket, we give the analytical solution to the atomic excitation, as well as the numerical results for transmitted and reflected light. We compare our time-dependent theory to the stationary limit, and we have reported that our time-dependent theory contains the stationary reflection and transmission spectra of earlier work by Shen and Fan [39]. To optimize the excitation probability of the emitter it is essential to choose incoming wavepackets of optimum duration. Within the class of Gaussian single-photon wavepackets, excitation efficiencies up to $40 \%$ are possible for emitters coupled to infinite waveguides. We further studied the impact of the finite bandwidth of the incoming wavepacket on the atomic excitation, and found the maximum excitation probablity to be $40 \%$. This high atomic excitation simply generated by the Gaussian distributed wavepacket is close to the fundamental limit of $50 \%$ set by reciprocity. In order to obtain an even higher excitation probability, we propose to engineer the photonic environment of the emitter to suppress the SE into all channels, except the one into which the incoming single photon is funneled. Practically, by terminating a plasmonic nanowire and positioning the emitter properly, we found that most of the light, up to $91 \%$, can be directed into a single channel. Reciprocity guarantees that if we use the high SE $\beta$-factor channel for pumping, a high atomic excitation in excess of 50\% can be achieved. Indeed, we found that a value of $72.4 \%$ can be achieved by using a very simple structure, i.e. a terminated metallic nanowire. This result is obtained with a Gaussian distributed single-photon wavepacket and can be improved by shaping the optical pulse. Using waveguide dispersion for pulse shaping, a further modest improvement can be achieved. 
As an outlook, we envisage that our time-dependent theory for the atomic excitation can be useful for the analysis of different experimentally relevant scenarios and for ongoing experimental activities that focus on coupling a freely propagating photon to an atom [13-15]. The relevance of engineering the photonic environment of the emitter is evident from our previous discussions for achieving a high atomic excitation rate, and might provide new guidelines to perform future experiments. Particularly promising for achieving high atomic excitation is the use of 3D photonic structures, i.e. optical nanoantennas [21, 32, 33], where the temporal and spatial coherence of the incoming wavepacket can be fully addressed. Optimizing single-photon absorption using the methods discussed in this paper will have a high impact throughout the photonics community, spanning from quantum optics, single-molecule absorption microscopy, to photovoltaics.

\section{Acknowledgments}

We thank Anders S Sørensen and Peter Lodahl for fruitful discussions. We also further thank Yves Rezus for a careful reading of the manuscript. YTC and MW acknowledge financial support from the Danish Research Council for Technology and Production Sciences (grant numbers 10093787 and 274070080, respectively). This work is part of the research program 'Stichting voor Fundamenteel Onderzoek der Materie (FOM)', which is financially supported by the 'Nederlandse Organisatie voor Wetenschappelijk Onderzoek (NWO)'. AFK was supported by a VIDI fellowship funded by NWO.

\section{Appendix A. Normalization in the continuous-mode quantization scheme}

In the well-known discrete-mode quantization scheme [51], the commutation rules for the photon operator obey $\left[\hat{a}_{\lambda}, \hat{a}_{\lambda^{\prime}}^{\dagger}\right]=\delta_{\lambda \lambda^{\prime}}$. The normalization condition for these discrete modes is $\int \varepsilon(\boldsymbol{r})\left[E_{\lambda}(\boldsymbol{r})\right] \cdot\left[E_{\lambda^{\prime}}(\boldsymbol{r})\right] \mathrm{d} V=\delta_{\beta, \beta^{\prime}} \delta_{p, p^{\prime}} \delta_{m, m^{\prime}} \delta_{q, q^{\prime}} N_{\mathrm{dis}}$. Accordingly, the single-photon field in the discrete-mode quantization scheme is obtained as $\boldsymbol{E}_{\lambda, 1, \text { dis }}(\boldsymbol{r})=\boldsymbol{E}_{\lambda}(\boldsymbol{r}) \sqrt{\frac{\hbar \omega}{2 N_{\text {dis }}}}$, where $\omega=$ $\sqrt{c^{2}\left(\beta^{2}+q^{2}\right)}$. In this paper, we deal with 1D waveguides and hence a quantization box with infinite extent parallel to the $z$-axis but with a finite cross-sectional area in the transverse plane. We extend equation (6) to the continuous-mode quantization scheme. The mode spacing along the $z$-direction, $\triangle \beta$, tends to zero as the quantization length along the $z$-axis approaches infinity. Therefore, the sum over all the modes $\sum_{\lambda}$ can be substituted by

$$
\sum_{\lambda} \longrightarrow \sum_{\{m, p, \beta, q\}} \longrightarrow \frac{1}{\triangle \beta} \int \mathrm{d} \beta \sum_{\kappa} \longrightarrow \frac{1}{\triangle \beta} \int \mathrm{d} \omega \frac{1}{v_{\mathrm{g}}(\omega)} \sum_{\kappa},
$$

where $\triangle \beta$ is the mode spacing along the $z$-direction. In this substitution rule, $\sum_{\kappa}$ represents the sum over all the modes with frequency $\Omega$. The normalization condition in the continuousmode quantization scheme needs to be modified to $\operatorname{read} \int \varepsilon(\boldsymbol{r})\left[E_{\lambda}(\boldsymbol{r})\right] \cdot\left[E_{\lambda^{\prime}}(\boldsymbol{r})\right] \mathrm{d} V=\delta(\beta-$ $\left.\beta^{\prime}\right) \delta_{p, p^{\prime}} \delta_{m, m^{\prime}} \delta_{q, q^{\prime}} N_{\text {con }}$. Accordingly, the single-photon field in the continuous-mode quantization scheme is obtained as

$$
\boldsymbol{E}_{\lambda, 1, \mathrm{con}}(\boldsymbol{r})=\boldsymbol{E}_{\lambda}(\boldsymbol{r}) \sqrt{\frac{\hbar \omega_{\lambda}}{2 N_{\mathrm{con}}}} .
$$


As a side remark, the discrete Kronecker delta and the continuous Dirac delta-function are related by $\delta_{\beta, \beta^{\prime}} \rightarrow \triangle \beta \delta\left(\beta-\beta^{\prime}\right)$, which indicates that the normalization factor has incorporated a factor of $\triangle \beta$ that yields the translation of the single-photon field from the discrete mode quantization to the continuous-mode quantization as $\boldsymbol{E}_{\lambda, 1, \text { con }}(\boldsymbol{r})=\boldsymbol{E}_{\lambda, 1, \mathrm{dis}}(\boldsymbol{r}) / \sqrt{\triangle \beta}$.

\section{Appendix B. Laplace transform method for a Gaussian input single-photon wavepacket}

An alternative approach to obtain the atomic excitation probability as a function of time that is distinct from our direct time integration of equation (8) to obtain equation (12) is to use a Laplace transform method reported by Wubs et al [52]. In this appendix, we summarize the Laplace method. By Laplace transforming Heisenberg's equation of motions of the atomic operator $\hat{b}(t)$ and field operator $\hat{a}(t)$, one can include the initial atomic excitations $\hat{b}(t=0)$ and photonic excitations $\hat{a}(t=0)$ in the dynamics. More details can be found in [52]. After making a pole approximation, the inverse Laplace transform gives the dynamical solution to the atomic operator,

$$
\begin{aligned}
\hat{b}(t)=\hat{b}(0) \mathrm{e}^{-\mathrm{i}\left(\Omega_{A}+\epsilon\right) t-\Gamma t / 2}+\frac{1}{\hbar} \int_{-\infty}^{-\infty} \mathrm{d} \omega^{\prime}\left[g\left(\omega^{\prime}\right) \hat{a}_{\left(\omega^{\prime},+\right)}(0)+g^{*}\left(\omega^{\prime}\right) \hat{a}_{\left(\omega^{\prime},-\right)}(0)\right] \\
\times \frac{\mathrm{e}^{-\mathrm{i} \omega^{\prime} t}-\mathrm{e}^{-\mathrm{i}\left(\Omega_{A}+\epsilon\right) t-\Gamma t / 2}}{\omega^{\prime}-\Omega_{A}-\epsilon+\mathrm{i} \Gamma / 2}
\end{aligned}
$$

where $\Gamma$ is the total SE decay rate, $\epsilon$ is the Lamb shift of atomic transition $\Omega_{A}$ of the emitter due to the coupling to the waveguide modes, $g\left(\omega^{\prime}\right)$ is the coupling strength, $\hat{b}(0)$ is the initial atomic excitation and $\hat{a}_{\left(\omega^{\prime},+\right)}(0)\left(\hat{a}_{\left(\omega^{\prime},+\right)}(0)\right)$ is the initial right (left) propagating optical excitation. The atomic population operator $\hat{N}_{A}(t)$ is given as $\left\langle\hat{N}_{A}(t)\right\rangle=\left\langle i\left|\hat{b}^{\dagger}(t) \hat{b}(t)\right| i\right\rangle$, with the initial condition given by $|i\rangle=|g\rangle \otimes \int \mathrm{d} \omega^{\prime} S\left(\omega^{\prime}\right) \hat{a}_{\left(\omega^{\prime},+\right)}(0)|0\rangle_{p}$, and $S\left(\omega^{\prime}\right)$ defines the incident single-photon wavepacket. Using the same Gaussian distributed single-photon wavepacket as the initial condition that is used in section 3, i.e. $S(k)=C_{k}^{g}(t=0)$, one finds that $|i\rangle=|g\rangle \otimes \int \mathrm{d} k S(k) a_{k,+}^{\dagger}|0\rangle$, which can be reformulated as an integral over frequency $|i\rangle=$ $|g\rangle \otimes \int \mathrm{d} \omega^{\prime} \chi\left(\omega^{\prime}\right) a_{\omega^{\prime},+}^{\dagger}|0\rangle$, with $\chi(\omega)=S\left(\frac{\omega}{v_{p}}\right) / \sqrt{v_{\mathrm{g}}(\omega)}, a_{\omega}^{\dagger}=a_{k}^{\dagger} / \sqrt{v_{\mathrm{g}}(\omega)}$. The group velocity and phase velocity are defined as $v_{\mathrm{g}}=\mathrm{d} \omega / \mathrm{d} k, v_{p}=\omega / k$. Within the linear dispersion approximation, $v_{\mathrm{g}}$ is equal to $v_{p}$. The expectation value of the excited atomic state reads as follows:

$$
\left\langle N_{A}(t)\right\rangle=\left|\int_{-\infty}^{\infty} \mathrm{d} \omega g_{\omega} \frac{\mathrm{e}^{-\mathrm{i} \omega t}-\mathrm{e}^{-\mathrm{i}\left(\omega_{A}+\epsilon\right) t-(\Gamma) t / 2}}{\omega-\Omega_{A}-\epsilon+\mathrm{i}(\Gamma) / 2} \chi(\omega)\right|^{2} .
$$

Using $\sigma=\omega-\omega_{0}$ and omitting the Lamb shift term $\epsilon$, one obtains

$$
\left\langle N_{A}(t)\right\rangle=\left|\left[\frac{2}{\pi \Omega^{2}}\right]^{\frac{1}{4}} \sqrt{\frac{\Gamma}{4 \pi}} \int_{-\infty}^{\infty} \mathrm{d} \sigma \frac{\mathrm{e}^{\left[-\mathrm{i} \sigma\left(t-t_{0}\right)-\frac{\sigma^{2}}{\Omega^{2}}\right]}-\mathrm{e}^{\left[-(\Gamma) t / 2+\mathrm{i} \sigma t_{0}-\frac{\sigma^{2}}{\Omega^{2}}\right]}}{\sigma+\mathrm{i} \Gamma / 2}\right|^{2} .
$$

By rewriting $\frac{1}{\sigma+\mathrm{i} \Gamma / 2}$ as $\frac{1}{\sigma+\mathrm{i} \Gamma / 2}=-\mathrm{i} \int_{0}^{\infty} \mathrm{d} \xi \mathrm{e}^{\mathrm{i}(\sigma+\mathrm{i} \Gamma / 2) \xi}$ and performing the $\sigma$-integral, one can further simplify $\left\langle N_{A}(t)\right\rangle$ as follows:

$\left\langle N_{A}(t)\right\rangle=\left|C_{0}^{\mathrm{e}}(t)\right|^{2}=\left|s \frac{1}{2} \sqrt{\frac{\pi}{a}} \mathrm{e}^{\left(b^{2} / a\right)-c}\left[\operatorname{erf}\left(\sqrt{a} t+\frac{b}{\sqrt{a}}\right)-\operatorname{erf}\left(\frac{b}{\sqrt{a}}\right)\right] \mathrm{e}^{-(\Gamma / 2) t}\right|^{2}$,

which is consistent with equation (12). 


\section{References}

[1] Raimond J M, Brune M and Haroche S 2007 Rev. Mod. Phys. 73565

[2] Gérard J M, Sermage B, Gayral B, Legrand B, Costard E and Thierry-Mieg V 1998 Phys. Rev. Lett. 811110

[3] Pelton M, Santori C, Vučković J, Zhang B, Solomon G S, Plant J and Yamamoto Y 2002 Phys. Rev. Lett. 89233602

[4] Wilk T, Webster S C, Kuhn A and Rempe G 2007 Science 317488

[5] Hennessy K, Badolato A, Winger M, Gerace D, Atatüre M, Fält S, Hu E L and Imamoğlu A 2007 Nature 445896

[6] Reithmaier J P, Sȩk G, Löffer A, Hofmann C, Kuhn S, Reitzenstein S, Keldysh L V, Kulakovshii V D, Reinecke T L and Forchel A 2004 Nature 432197

[7] O’Brien J L, Furusawa A and Vučković J 2009 Nat. Photonics 3689

[8] Kimble H J 2008 Nature 4531023

[9] Schoelkopf R J and Girvin S M 2008 Nature 451664

[10] Cirac J I and Zoller P 2007 Phys. Rev. Lett. 744091

[11] Cirac J I, Zoller P, Kimble H J and Mabuchi H 1997 Phys. Rev. Lett. 783221

[12] Julsgaard B, Sherson J, Cirac J I, Fiurášek J and Polzik E S 2004 Nature 432482

[13] Wrigge G, Gerhardt I, Hwang J, Zumofen G and Sandoghdar V 2008 Nat. Phys. 460

[14] Lindlein N, Maiwald R, Konermann H, Sondermann M, Peschel U and Leuchs G 2007 Laser Phys. 17927

[15] Piro N, Rohde F, Schuck C, Almendros M, Ghosh J, Haase A, Hennrich M, Dubin F and Eschner J 2011 Nat. Phys. 717

[16] van Enk S J 2004 Phys. Rev. A 69043813

[17] Sondermann M, Maiwald R, Konermann H, Lindlein N, Peschel U and Leuchs G 2007 Appl. Phys. B 89489

[18] Manga Rao V S C and Hughes S 2007 Phys. Rev. Lett. 99193901

[19] Gerhardt I, Wrigge G, Bushev P, Zumofen G, Agio M, Pfab R and Sandoghdar V 2007 Phys. Rev. Lett. 98033601

[20] Zumofen G, Mojarad N M, Sandoghdar V and Agio M 2007 Phys. Rev. Lett. 101180404

[21] Kühn S, Håkanson U, Rogobete L and Sandoghdar V 2006 Phys. Rev. Lett. 97017402

[22] Chang D E, Sørensen A S, Hemmer P R and Lukin M D 2007 Nat. Phys. 3807

[23] Stobińska M, Alber G and Leuchs G 2009 Europhys. Lett. 8614007

[24] Wallraff A, Schuster D I, Blais A, Frunzio L, Huang R S, Majer J, Kumar S, Girvin S M and Schoelkopf R J 2004 Nature 431162

[25] Blais A, Huang R S, Wallraff A, Girvin S M and Schoelkopf R J 2004 Phys. Rev. A 69062320

[26] Fan S, Kocabaş Ş E and Shen J T 2010 Phys. Rev. A 82063821

[27] Kukura P, Celebrano M, Renn A and Sandoghdar V 2009 Nano Lett. 9926

[28] Gaiduk A, Yorulmaz M, Ruijgrok P V and Orrit M 2010 Science 330353

[29] Akimov A V, Mukherjee A, Yu C L, Chang D E, Zibrov A S, Hemmer P R, Park H and Lukin M D 2007 Nature 450402

[30] Lund-Hansen T, Stobbe S, Julsgaard B, Thyrrestrup H, Sünner T, Kamp M, Forchel A and Lodahl P 2008 Phys. Rev. Lett. 101113903

[31] Koenderink A F 2009 Nano Lett. 94228

[32] Curto A G, Volpe G, Taminiau T H, Kreuzer M P, Quidant R and van Hulst N F 2010 Science 329930

[33] Kosako T, Kadoya Y and Hofmann H F 2010 Nat. Photonics 4312

[34] Chen Y, Lodahl P and Koenderink A F 2010 Phys. Rev. B 82081402

[35] Chang D E , Sørensen A S, Hemmer P R and Lukin M D 2006 Phys. Rev. Lett. 97053002

[36] Jun Y C, Kekapture R D, White J S and Brongersma M L 2008 Phys. Rev. B 78153111

[37] Lecamp G, Lalanne P and Hugonin J P 2007 Phys. Rev. Lett. 99023902

[38] Chen Y, Nielsen T R, Gregersen N, Lodahl P and Mørk J 2010 Phys. Rev. B 81125431

[39] Shen J T and Fan S 2005 Opt. Lett. 302001 
[40] Heugel S, Villar A S, Sondermann M, Peschel U and Leuchs G 2010 Laser Phys. 1100

[41] Witthaut D and Sørensen A S 2010 New J. Phys. 12043052

[42] de Vries P, van Coevorden D V and Lagendijk A 1998 Rev. Mod. Phys. 70447

[43] Gorshkov A V, André A, Fleischhauer M, Sørensen A S and Lukin M D 2007 Phys. Rev. Lett. 98123601

[44] Rephaeli E, Shen J T and Fan S 2010 Phys. Rev. A 82033804

[45] Kolchin P, Belthangady C, Du S, Yin G Y and Harris S E 2008 Phys. Rev. Lett. 101103601

[46] Dorner U and Zoller P 2002 Phys. Rev. A 66023816

[47] Domokos P, Horak P and Ritsch H 2002 Phys. Rev. A 65033832

[48] Kien F L, Balykin V I and Hakuta K 2006 Phys. Rev. A 73013819

[49] Rist S, Eschner J, Hennrich M and Morigi G 2008 Phys. Rev. A 78013808

[50] Coldren L A and Corzine S W 1995 Diode Lasers and Photonic Integrated Circuits (New York: WileyInterscience)

[51] Loudon R 2000 The Quantum Theory of Light (New York: Oxford University Press)

[52] Wubs M, Suttorp L G and Lagendijk A 2004 Phys. Rev. A 70053823

[53] Drexhage K H 1970 J. Lumin. 1-2 693

[54] Chen Y, Gregersen N, Nielsen T R, Mørk J and Lodahl P 2010 Opt. Express 1812489

[55] Miyagi M and Nishida S 1979 Appl. Opt. 18678 